\title{
Viveros escolares como estrategia pedagógica para la recuperación de la ribera del río Magdalena ${ }^{1}$
}

\section{School plant nurseries as pedagogic strategy and conservation of the Magdalena river}

DOI: http://dx.doi.org/10.17981/cultedusoc.9.3.2018.89

Artículo de investigación. Fecha de recepción: 15/06/2018. Fecha de aceptación: 27/11/2018

Guillermo Varela Villarruel'2;

Erladis Quiroz González; Nivia Vásquez Viadero; Gustavo Jacome Centeno; Gladys Alvear Ospino; Edwin Pérez Moreno y César Álvarez Sánchez ${ }^{3}$

Institución Educativa Departamental Arcesio Caliz Amador, sede Francisco José de Caldas (Colombia) arseciocalizinst@gmail.com

Para citar este artículo:

Varela, G., Quiroz, E., Vásquez, N., Jacome, G., Alvear, G., Pérez, E. y Álvarez, C. (2018). Viveros escolares como estrategia pedagógica para la recuperación de la ribera del río Magdalena. Cultura. Educación y Sociedad 9(3), 755-762. DOI: http://dx.doi.org/10.17981/ cultedusoc.9.3.2018.89

\section{Resumen}

El cambio climático y sus efectos meteorológicos extremos como sequias e inundaciones afectan todo el planeta. Precisamente, las inundaciones son el principal problema sobre poblaciones ribereñas. En el Banco Magdalena a orilla del río Magdalena la deforestación de la ribera provocó la erosión de esta. Desde la Escuela Urbana Francisco José de Caldas se planteó el establecimiento de un vivero como alternativa de reforestación y estrategia pedagógica para generar un comportamiento proambiental en la comunidad. A través de encuestas se evaluó el nivel de conocimiento de la comunidad escolar sobre la deforestación y la erosión de la ribera, así como el nivel de compromiso para desarrollar y mantener el proyecto de vivero, para ello se definieron siete especies de plantas nativas. El proyecto permitió una transversalidad del currículo, una motivación y enseñanza de valores en los estudiantes y una sensibilización en la comunidad en el área de influencia.

Palabras clave: Ribera, erosión, vivero escolar, reforestación.

\section{Abstract}

Climate change and its extreme weather effects such as droughts and floods affect the entire planet. Precisely the floods are the main problem on riparian populations. In the municipality of $\mathrm{El}$ Banco (Magdalena), on the banks of the Magdalena River, deforestation of the river bank caused its erosion. The "Escuela Urbana Francisco José de Caldas" proposed the establishment of a nursery plant as an alternative of reforestation and pedagogical strategy to generate a pro-environmental behavior in the community. Through surveys, the level of knowledge of the school community about deforestation and riparian erosion was evaluated, as well as the level of commitment to develop and maintain the nursery; seven species of native plants were defined for planting in the nursery. The project allowed a transversality of the curriculum, promoted motivation and teaching in human values in the students and an awareness in the community in the area of influence.

Keywords: Riverbank, erosion, school nursery, reforestation.

1 Este artículo ha sido derivado del Programa de Fortalecimiento de la Cultura Ciudadana y Democrática CT+I a través de la IEP apoyada en TIC en el Departamento de Magdalena: CICLON

2 Líder del Grupo de investigación "Protectores de la Ribera".

3 Docentes de la institución Educativa Departamental Arcesio Caliz Amador, sede Francisco José de Caldas.

- The author; licensee Universidad de la Costa - CUC.

Cultura, Educación y Sociedad vol. 9 no. 3, pp. 755-762. Diciembre, 2018

Barranquilla. ISSN 2389-7724 Online 


\section{Introducción}

El ser humano a lo largo de la historia ha transformado su entorno dependiendo de sus necesidad e intereses, actualmente una de las principales problemáticas que enfrentamos como especie es el calentamiento global que genera problemas sociales, económicos y ambientales, e impacta indirectamente en otros aspectos de nuestras vidas (Cortés, 2011), por consiguiente, es necesario un cambio en la manera como nos relacionamos con el medio y es de suma importancia como se aborda esta relación desde las escuelas, a través del fomento de un comportamiento proambiental, entendido este como: "El conjunto de acciones intencionales, dirigidas y efectivas que responden a requerimientos sociales e individuales y que resultan en la protección del medio" (Corral Verdugo, 2000; Martínez-Soto, 2006; citados en Cortés, 2011), y definiendo actividades en el marco del desarrollo sostenible (Couldrey y Herson, 2008; Cortés, 2011; Cortés-Peña, 2016).

Las riberas de los ríos han sido desde la antigüedad sitios propicios para asentamientos humanos. Importantes centros urbanos del mundo se encuentran ubicados a orillas de grandes ríos (e.g. Paris, Londres, Hamburgo, Budapest y otras en Europa, Buenos Aires, Montevideo y Manaos en Suramérica). En el Caribe colombiano encontramos ciudades como Barranquilla, Montería, Valledupar, y el municipio de El Banco Magdalena, entre otras (Hernández, Herrera, Villalba y Gómez, 2007).

Dentro de las actividades del hombre ribereño, además de la obvia actividad pesquera, se destaca el uso del suelo para agricultura y ganadería; siendo estas dos últimas, las que mayor impacto generan en el medio, principalmente por el manejo inadecuado de las tierras y la escasa mano de obra tecnificada, lo que ha llevado a la tala y quema de bosques, sumado a pocos procesos de reforestación y a un mal manejo de residuos sólidos, que en muchos casos son fuente de contaminación de los cuerpos de agua (Guidi y Iglesias, 2015). La tala indiscriminada puede generar erosión de las riberas por degradación del suelo y sumado a la sedimentación del lecho de los ríos, pueden ocasionar serios problemas ambientales para las comunidades que los habitan, como las inundaciones (Benavides y León 2007, Morales 2016) y (Organización de las Naciones Unidas, 2015).

El municipio de el Banco Magdalena, se encuentra a orillas del brazo de Mompox del río Magdalena, pobladores de barrios como La Playa, La Candelaria, Dos de Febrero, entre otros, sufren continuamente de los estragos de las crecientes del río Magdalena; estas comunidades se dedican a la pequeña agricultura de pancoger (i.e. yuca, guineos, mafufos, plátanos, ahuyamas, habichuelas, mangos, patillas, melones, etc.), siendo para estas familias su principal sustento y base económica.

El Banco es uno de los municipios del Departamento del Magdalena con mayor susceptibilidad a inundaciones (CORPOICA, 2018), por lo que las poblaciones ribereñas se han adaptado a convivir con la dinámica del río, sus causas y consecuencias. Además, la falta de defensa natural en las riveras trae como consecuencia desprendimientos de las orillas y pérdida de terrenos para las actividades pecuarias de sus habitantes.

La escuela como institución de formación debe contribuir a la solución de los diferentes problemas de su entorno, especialmente a las comunidades en su área de influencia. Una realidad en las 
instituciones educativas del municipio de El Banco es el desinterés por parte de los estudiantes en cuanto a la problemática ambiental, reflejado en el nivel de aseo de los espacios, poco cuidado y desconocimiento del medio ambiente y la vegetación. A través del quehacer educativo en la Escuela Urbana Francisco José de Caldas se planteó la necesidad de fomentar espacios de formación y sensibilización ambiental y el fomento del comportamiento proambiental en estudiantes de primera edad, esto a través del diseño de un vivero escolar ${ }^{4}$ de plantas nativas y adaptadas a las riberas de los ríos, en donde se vincule toda la comunidad escolar, que además de servir como estrategia pedagógica, ayudará a mitigar el problema de deforestación que afecta a las comunidades de los La Candelaria y La Playa, en el municipio de El Banco, Magdalena.

\section{Metodología}

El enfoque de esta investigación tiene su origen epistemológico en el conocimiento del entorno social del hombre, por lo tanto parte del trabajo se centró en definir el estado del conocimiento por parte de la comunidad educativa y en el área de influencia directa de la misma. Se diseñaron 3 encuestas destinadas específicamente para a) estudiantes, b) padres de familia y c) docentes y directivos de la Escuela Urbana Francisco José de Caldas de la Institución Educativa Departamental Arcesio Cáliz Amador del Municipio de El Banco Magdalena, donde se indagó sobre el conocimiento del estado de la ribera, el impacto sobre la

4 Vivero: lugar donde se cultivan arboles u otras plantas, y donde pueden existir una o más especies en germinación, crecimiento hasta cierto tamaño para luego ser trasplantado a un sitio determinado con características propias aptas para el desarrollo total de las plantas (Trujillo, 2014) misma y la pertinencia de establecer un vivero escolar. De las 200 personas que conforman la comunidad educativa 33 fueron encuestadas.

Adicionalmente para recolectar la información sobre las especies aptas para establecer el vivero escolar se realizó observación directa y salidas de campo con los estudiantes a los sitios de los barrios de La Playa y la Candelaria, donde se realizaron adicionalmente entrevistas a estudiantes, padres de familias y vecinos del sector.

\section{Resultados}

\section{Análisis de las Encuestas por población}

\section{- Estudiantes}

Dentro del sistema de encuestas realizado a la comunidad de estudiantes de la Escuela Urbana Francisco José de Caldas, del municipio de El Banco, Magdalena, se pudo determinar que los estudiantes en general tienen destreza en la identificación de sitios de ribera y zonas donde se presenta erosión, así como las causas de este fenómeno y como evitarlo. Los alumnos muestran conciencia acerca de la problemática ambiental e interés es su restauración y protección apoyando la iniciativa de crear un vivero dentro de la institución, mostrándose además entusiasmados y haciéndose responsables con la organización y mantenimiento de este; para lo cual es importante incentivar y desarrollar en el estudiantado conceptos previos sobre el funcionamiento de un sistema de viveros y las características propias de las especies que forman parte de una ribera para así descartar falencias y hacer del vivero un sistema sostenible. 


\section{- Padres de familia}

Con base en el sistema de encuestas realizado a los padres de familia de la comunidad, fue evidente la motivación y disposición de la mayoría de los padres en la participación propia y de sus hijos en el proyecto de vivero en la Escuela Urbana Francisco José de Caldas. La mayoría de los padres afirmaron tener conocimiento de la creciente erosión en las riberas aledañas al municipio y su impacto en la comunidad. Manifestaron su compromiso con la donación de semillas y tallos de especies nativas la creación de un semillero escolar que permita reforestar las zonas calificadas en estado críticos y así contribuir al cuidado del medio. Manifestaron, además, que es importante para que las actividades sean llevadas a cabo con éxito, vincular estrategias que motiven a la participación y el compromiso de todos.

\section{- Docentes}

Partiendo de la indagatoria realizada a docentes y directivos, a pesar de la poca experiencia en la temática para algunos, los docentes se mostraron motivados y comprometidos con la realización de este proyecto, quienes lo consideran una herramienta pedagógica para generar y fomentar en el estudiantado valores como la responsabilidad, trabajo en equipo, sensibilización y respeto ambiental y la toma de decisiones e iniciativas propias, además que el proyecto puede servir como eje transversal en las diferentes asignaturas para la formación académica de los estudiantes. Los docentes y directivos expresaron su disposición en integrar el proyecto vivero escolar como proyecto de aula según su área, destacan la importan- cia de la participación de los padres de familia y se comprometieron a implementar asambleas periódicas para planificar en acompañamiento con los distintos participes el desarrollo de este proyecto.

\section{- Vivero escolar}

Resultado de la indagación con la comunidad se seleccionaron algunas especies nativas de la región (tabla 1 ), que se caracterizan por sus adaptaciones a las condiciones de la ribera y que pueden ayudar a la recuperación de esta, ya que son identificadas por los pobladores como especies con raíces profundas que ofrecen sostén al terreno, lo que les permite soportar el embate de las aguas del río Magdalena; además, como especies con gran follaje que pueden aportar sombra. Todas estas especies de árboles han germinado en el vivero escolar, donde se mantienen en bolsas y luego son trasplantadas en bolsas rellenas de abonos a su lugar de sembradíos.

\section{Discusión}

La comunidad educativa de la Escuela Urbana Francisco José de Caldas del Municipio de El Banco Magdalena es la primera entidad institucional de carácter educativo que lidera una campaña ambiental para reforestar las riberas del río en el municipio, específicamente en los barrios La Playa y La Candelaria con el objetivo de mitigar la continua erosión de los ríos, a través de la siembra de árboles nativos con adaptaciones propias a las riberas se puede solucionar una problemática que afecta a los habitantes de la región. 


\section{TABLA 1}

Especies de plantas seleccionadas para sembrar en el vivero escolar.

\begin{tabular}{|c|c|c|c|c|}
\hline Familia & Especie & $\begin{array}{l}\text { Nombre } \\
\text { Común }\end{array}$ & $\begin{array}{c}\text { Usos y } \\
\text { observaciones }\end{array}$ & Hábitos \\
\hline Euphorbiaceae. & Hura Crepitans L. & $\begin{array}{l}\text { Ceiba } \\
\text { amarilla. }\end{array}$ & $\begin{array}{l}\text { Ebanistería y } \\
\text { artesanías. }\end{array}$ & $\begin{array}{l}\text { Árbol gigantesco de } \\
\text { tierras cálidas, con látex } \\
\text { tóxico, tronco cubierto de } \\
\text { espinas cónicas leñosas. } \\
\text { Frutos redondos y } \\
\text { achatados. }\end{array}$ \\
\hline Fabaceae. & $\begin{array}{l}\text { Pithecellobium } \\
\text { lanceolatum } \\
\text { (Humb. \& Bonpl. } \\
\text { ex Willd.) Benth. }\end{array}$ & Pintacanilla. & $\begin{array}{l}\text { Refugio para } \\
\text { el ganado, leña } \\
\text { combustible, } \\
\text { usos medicinales } \\
\text { y extracción } \\
\text { de taninos. } \\
\text { Localmente } \\
\text { como pintura } \\
\text { para esteras }\end{array}$ & $\begin{array}{l}\text { Árbol de bosques } \\
\text { húmedos y secos, de } \\
\text { hasta } 12 \text { m de alto, } \\
\text { flores blancas y frutos } \\
\text { curvados de color rojizo. }\end{array}$ \\
\hline Moraceae. & $\begin{array}{l}\text { Ficus dendrocida } \\
\text { Kunth. }\end{array}$ & Suan. & $\begin{array}{l}\text { Leña, } \\
\text { combustible. }\end{array}$ & $\begin{array}{l}\text { Árbol de tierras } \\
\text { templadas, comienzan } \\
\text { su vida creciendo sobre } \\
\text { otros árboles y a medida } \\
\text { que extienden sus raíces } \\
\text { hacia el suelo envuelve } \\
\text { con ellas al árbol } \\
\text { huésped. }\end{array}$ \\
\hline Poaceae. & $\begin{array}{l}\text { Guadua } \\
\text { amplexifolia J. } \\
\text { Presl. }\end{array}$ & $\begin{array}{l}\text { Bambú } \\
\text { criollo. }\end{array}$ & $\begin{array}{l}\text { Construcción, } \\
\text { elaboración de } \\
\text { utensilios y } \\
\text { artesanías. }\end{array}$ & $\begin{array}{l}\text { Bambú de gran porte } \\
\text { de tierras cálidas y } \\
\text { templadas, forma } \\
\text { extensos grupos, } \\
\text { principalmente cerca } \\
\text { en los cursos de agua } \\
\text { brindándoles protección. }\end{array}$ \\
\hline Polygonaceae. & $\begin{array}{l}\text { Coccoloba } \\
\text { acuminata Kunth. }\end{array}$ & Maizcocho. & $\begin{array}{l}\text { Leña, postes } \\
\text { para cercas, } \\
\text { uso ornamental } \\
\text { y frutos } \\
\text { comestibles. }\end{array}$ & $\begin{array}{l}\text { Arbolito de tierras } \\
\text { cálidas, de hasta } 10 \mathrm{~m} \\
\text { de alto, hojas gruesas } \\
\text { y quebradizas. Frutos } \\
\text { carnosos apiñados en } \\
\text { forma de racimos. }\end{array}$ \\
\hline Polygonaceae. & $\begin{array}{l}\text { Coccoloba uvifera } \\
\text { (L.) L. }\end{array}$ & Uvero. & $\begin{array}{l}\text { Barrera contra } \\
\text { el viendo } \\
\text { en costas, } \\
\text { leña, frutas } \\
\text { comestibles, usos } \\
\text { medicinales y } \\
\text { extracción de } \\
\text { taninos. }\end{array}$ & $\begin{array}{l}\text { Árbol de pequeño y } \\
\text { mediano porte de tierras } \\
\text { cálidas, tolerante a la } \\
\text { sal, crece en sustratos } \\
\text { rocos. Frutos en racimos } \\
\text { con forma de uva. }\end{array}$ \\
\hline Polygonaceae. & $\begin{array}{l}\text { Symmeria } \\
\text { paniculata Benth. }\end{array}$ & Mangle. & $\begin{array}{l}\text { Resistencia a las } \\
\text { inundaciones. }\end{array}$ & $\begin{array}{l}\text { Árbol de tierras bajas } \\
\text { tropicales, crece a orillas } \\
\text { de las cuencas, tolerante } \\
\text { a la sal. }\end{array}$ \\
\hline
\end{tabular}

Fuente: Instituto de Ciencias Naturales (2004). 
Con la organización del vivero escolar se observó el interés en los estudiantes, se consiguió generar valores como: responsabilidad, cooperación, puntualidad, cuidado del medio ambiente, capacidad crítica, y espíritu de participación en la toma de decisiones para enfrentar y solucionar un problema, en ese sentido el vivero escolar sirvió como detonante para este cambio de actitud.

Por otro lado, la vinculación de todos los actores de la comunidad educativa interesadas en la problemática facilita la toma de decisiones y satisface el sentir de los inmersos en el proyecto. Los docentes desde su labor pedagógica lograron involucrar este proyecto en las diversas áreas del saber, desde el grado preescolar hasta el grado quinto se adecuó las temáticas según los estándares, objetivos de los grados e intereses de los estudiantes; así, por ejemplo:

a. En el área de matemáticas se fomentó y ayudó en el uso de la estadística, toma y análisis de datos, planos geométricos, conteos de plantas, concepto de medidas, pesos, y volúmenes de abonos.

b. En el área de la humanidades se utilizó el tema como estrategia de enseñanza de habilidades comunicativas como la relatoría, escritura, composiciones y temas de paneles y discusiones literarias, las coplas, adivinanzas, refranes, cuentos, narraciones, juegos de letras, silabas y demás estrategias según el grado y nivel de los estudiantes.

c. En el área de ciencias de la naturaleza y la ecología facilitó sensibilizar sobre el cuidado y conservación del medio ambiente, botánica básica, se motivó el interés por la investigación, participación, y dominio de temas de la vida diaria del hombre y su relación con el medio. d. En las áreas de educación religiosa, ética y moral se generó la admiración por la creación, el respeto y cuidado por el medio y por sus semejantes, el fomento de valores, todos estos aspectos positivos para la formación humana.

e. En el área de ciencias sociales para afianzar conocimientos del entorno social, modos de vida del hombre e interacción del mismo con el medio y como lo modifica, economías de los hombres de la ribera y sus costumbres, además de conocer las dificultades y problemáticas sociales del entorno y reflexionar sobre el planteamiento de soluciones a estas.

f. En educación física y recreación el realizar actividades en espacios abiertos del entorno escolar sirve para estimular y motivar a los estudiantes a relacionarse con la naturaleza y a tomarle gusto a las actividades lúdicas.

g. En las áreas de la informática y la tecnología ayuda al usar las TIC en el desarrollo de sus investigaciones y consultas, tomas de datos, y elaboración de informes, a través del uso de elementos como las tabletas, portátiles y computadoras para registrar sus experiencias.

h. En el área de educación artística el modelado con figuras, uso de materiales naturales como hojas, pedazos de cortezas de árboles, y demás elementos del medio en la elaboración de talleres para la elaboración de pequeñas artesanías, lo que fomenta la creatividad de los estudiantes. 
Además de todas estas motivaciones encontradas en el proceso del desarrollo de este proyecto de investigación, se logra el establecimiento de canales de comunicación con otras instituciones educativas, gubernamentales municipales, regionales y nacionales relacionadas con la temática de la reforestación y recuperación de zonas intervenidas y afectadas por la actividad del hombre; se vinculan medios de comunicación de prensa hablada y escrita a nivel local y regional y se visibiliza las problemáticas del río.

A largo plazo esperamos que el proyecto contribuya además a mejorar la temperatura en la zona, y que la comunidad se apersone del cuidado y mantenimiento de las plantas, con el apropiado seguimiento por parte de los estudiantes y docentes.

\section{Referencias}

CORPOICA (2018) Sistema Experto M.A.P.A.: Modelo de Adaptación y prevención Agroclimática. Recuperado de http:/www.corpoica.org.co:8086/ NetCorpoicaMVC/SEMapa con Acceso 18 julio 2018

Benavides, H., y León, G. (2007). Información técnica sobre gases de efecto invernadero y el cambio climático. Instituto de Hidrología, Meteorología y Estudios Ambientales - IDEAM. Recuperado de http://www.ideam.gov.co/ documents/21021/21138/Gases+de+Ef ecto+Invernadero+y+el+Cambio+Cli matico.pdf/7fabbbd2-9300-4280-befec11cf15f06dd

Cortés, O. (2011). Comportamiento proambiental y pensamiento económico en la construcción del desarrollo sostenible. Cultura Educación y Sociedad, 2(1), 43-56. Recuperado de https://revistascientificas.cuc.edu.co/ culturaeducacionysociedad/article/ view/931/pdf_152
Cortés-Peña, O. (2016). El desarrollo sostenible en relación sinérgica con el comportamiento pro ambiental y el comercio justo. International Journal of Management Science \& Operation Research, 1(1), 54-58

Couldrey, E. y Herson, M. (edit.) (2008). Cambio climático y desplazamiento. Revista migraciones forzadas, 31. Recuperado de http://www.fmreview. org/sites/fmr/files/FMRdownloads/ es/pdf/RMF31/RMF31.pdf

García, R. y Bolívar, R. (2017). Caracterización hidrométrica de las arcillas utilizadas en la fabricación de productos cerámicos en Ocaña, Norte de Santander. INGE CUC, 13(1), 53-60. https://doi.org/10.17981/ingecuc.13.1.2017.05

Guidi, G. y Iglesias, A. (2015). Turismo \& Universidad. Cultura, economía y sociedad. Avellaneda: Undav Ediciones.

Hernández, M., Herrera, R., Villalba, L. y Gómez, J. (2007). Estudio de caracterización transporte acuático. Recuperadodehttps://issuu.com/camaracci/ docs/sistema_ferreo_nacional._transporte

Organización de las Naciones Unidas. (2015). El suelo es un recurso no renovable. Su conservación es esencial para la seguridad alimentaria $y$ nuestro futuro sostenible. Recuperado de http://www.fao.org/3/a-i4373s. pdf

Strack, J., Suárez, J., Di Mauro, G. y Jacob, S. (2014). Impacto de la iluminación residencial eficiente en la calidad de la energía de una red de distribución. INGE CUC, 10(2), 9-19. Recuperado de https://revistascientificas.cuc.edu.co/index.php/ingecuc/ article/view/485 
UNESCO. (1980). El correo de la unesco.

Recuperado de http://unesdoc.unesco. org/images/0007/000747/074757so.pdf 